\title{
Effect of Double Suprarenalectomy on the Fatigability of the Nerve-Muscle Preparation in situ.
}

\author{
By \\ Hatiro Sibuta.

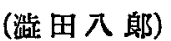 \\ (From the Physiological Laboratory of Professor Y. Satake, \\ Tohoku Teikoku Daigaku, Sendai.)
}

Studies rê the question whether or not double removal of the suprarenal capsules has some bearings upon the development of fatigue in animals is to be classified into three groups, namely (1) studies of the fatigability of the animal as the whole, or of the voluntary activity, (2) that of muscle on exciting it through its nerve, or on the indirect stimulation, and (3) that on the direct stimulation.

.In studying the question it is rather superfluous to say here that utmost care should be paid in order to avoid any mingling of influence of the operation itself besides that of the loss of the suprarenal capsules itself upon the experimental results.

With this kind of precautions Shoz $\mathbf{i}^{13}$ was able in the tread mill experiment to see the equality in the velocity with which the fatigue develops between the normal rabbit and the doubly decapsulated and indefinitely well living one.

It is then a further question whether or not a similar outcome will be obtainable if a nerve-muscle preparation in situ will be stimulated indirectly or directly instead of the tread mill experiment.

While all the reports ${ }^{2-13)}$ agree in noting a remarkable inferiority of the work capacity of the muscle preparation such as the gastrocnemic in rats or guinea-pigs doubly decapsulated or even unilaterally operated on, they are based upon the experiments carried out immediately after the decapsulation or a few days or weeks later. It is unquestionable that most of the animals were suffering from some weakness.

In the present work, which was carried out about five years ago, was used the rabbit, the animal which possesses invariably accessory cortical 
tissue, is capable of surviving indefinitely double removal of suprarenals if properly operated, ${ }^{14)}$ and really proved by $S h o \mathrm{ji}^{1)}$ of non-inferiority of the work capacity of doubly decapsulated, indefinitely surviving ones to the normal controls in the tread mill experiment, as above referred to. The latter work is fairly comparable to the present, given in the following pages.

\section{METHODS.}

Male and female rabbits of good nutrition were experimented on, without giving any narcosis throughout.

The peripheral cut end of the sciatic nerve was stimulated electrically, and the contraction of the gastrocnemius muscle was recorded, the calcaneus tendon being attached to the lever. The muscle was afterloaded with a lead weight of 50-100 grms.

All the arrangement and the operative procedure of exposing the nerve and muscle, inserting drills and fixing the animal were carried out in the manner much practised in the laboratory of Professor C. S. Sherrington, such as described in his text-book of practical exercise. ${ }^{16)}$ The Sherrington glass-sheathed electrode was used in an earlier form. ${ }^{16)}$ The stimulation was given automatically at 5 second intervals by means of a faradic current, using break shocks only. In the primary circuit of the induction apparatus a battery of 2 volts and a meteronom were placed, and in the secondary a rectifier, a condenser of $2 \mu \mathrm{F}$ and a Sherring ton electrode.

Registration of muscle contractions on a slow revolving extenson kymograph was made from time to time, the intervals being taken properly; when thought necessary it was done without interruption for some length of time. The total amount of work done was calculated by multiplying the sammeted height of the contraction by the weight lifted.

\section{DATA.}

3 normal rabbits and 7 doubly suprarenalectomized, long surviving ones were used in the fatigue experiment of the ischiadicus-gastrocnemius preparation in situ. The results are given graphically below.

As well known, the body temperature of rabbits falls, if they are fastened on an experiment table, for example. When rabbits, 4 in number, were fastened in the supine position for $8 \mathrm{hrs}$, the length of time in which the nerve-muscle preparation in situ of the doubly decapsulated rabbits becomes incapable of reacting against the electric stimulant, the body temperature fell by $\mathrm{r} .8-3.4^{\circ}$ in $3-5 \mathrm{hrs}$., followed by a very slow recovery. The room temperature was there $18-23^{\circ}$.

In the fatigue experiment this fall was guarded against properly by heating electrically the upper copper plate of the experiment table. The fall in the body 
temperature as well as overheating may interfere with the results in such a kind of experiment.

In the figure the work capacity is given on the ordinate with grms. $\mathrm{cm}$. and the time on the abscissa with hours. The capacities from the normal, control rabbits are given with light circles connected with dash lines and those from the decapsulated with heavy circles with solid lines. The circles are those actually determined. When those determined successively were different from each other, the lines are drawn as passing the mean value, indicated by a small circle.

Some words may be spent for the experiments in which some peculiarities were observed. Rabbit L: At terminal $(+)$, the gastrocnemius contracted only occasionally, but on stronger stimuli it was capable of responding with 744 grms. cm., but ten minutes later no more response. Rabbit M : At the

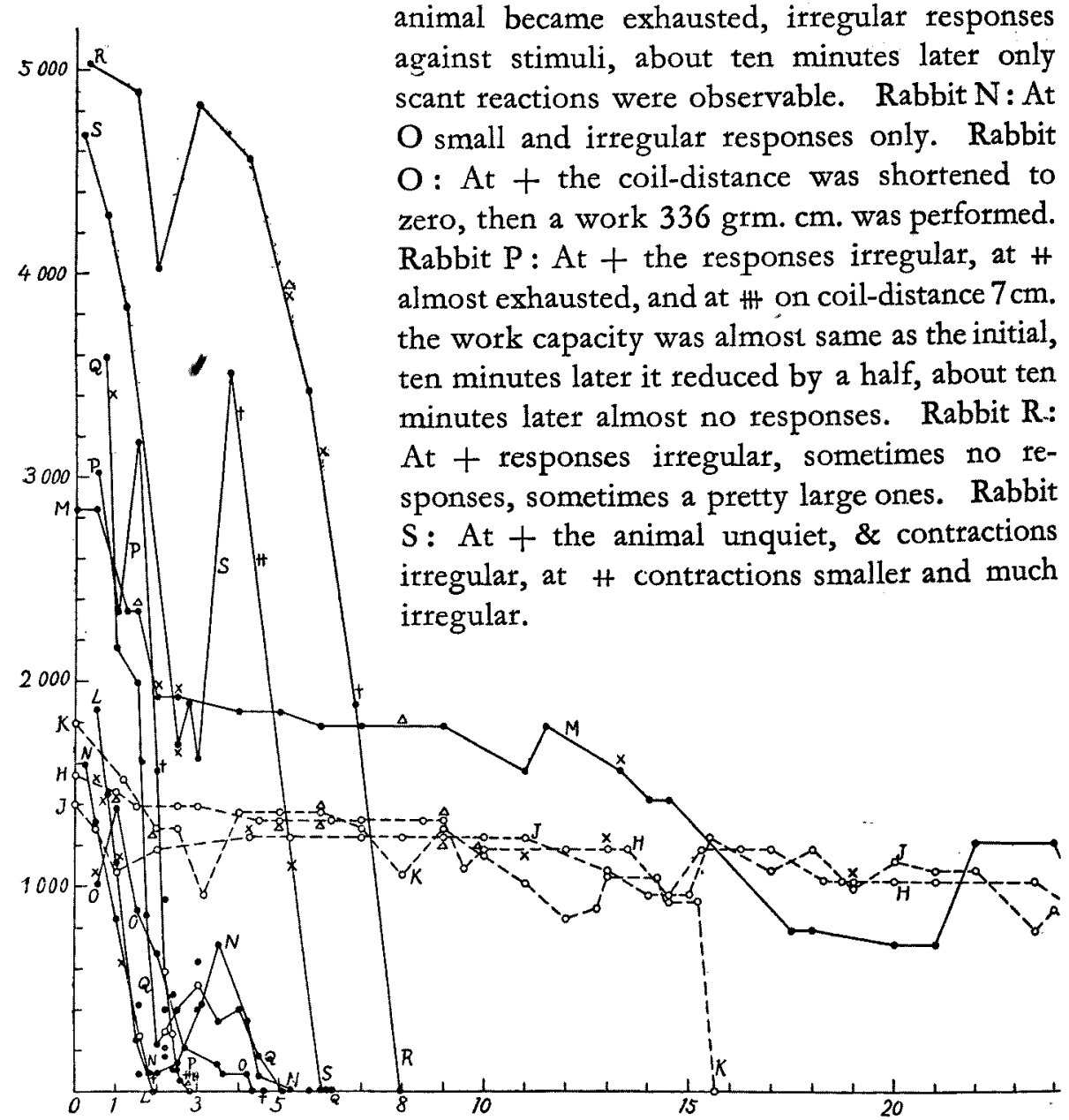


Lead weight: For normal animals :-H $730 \mathrm{~g}, \mathrm{~J} 560 \mathrm{~g}, \mathrm{~K} 560 \mathrm{~g}$. For decapsulated animals :-L $620 \mathrm{~g}, \mathrm{M} 710 \mathrm{~g}, \mathrm{~N} 420 \mathrm{~g}, \mathrm{O} 420 \mathrm{~g}, \mathrm{P} 520 \mathrm{~g}, \mathrm{Q} 570 \mathrm{~g}$, R $670 \mathrm{~g}, \mathrm{~S} 650 \mathrm{~g}$.

In none of the decapsulated rabbits, was found at autopsy any fragment of both the suprarenal glands. The accessory cortical tissues found on that occasion were as follows: Rabbit $\mathrm{L}$ ㅇ : $1 \times 0.9 \mathrm{~mm}$. on inf. cava vein below $\mathrm{R}$ liver, $2 \times 1.5 \mathrm{~mm}$. \& $1.5 \times 1.0 \mathrm{~mm}$. on inf. cava v., neat $R$ renal vessels. $0.7 \times 0.8 \mathrm{~mm}$. on $R$ ovary. Rabbit $M$ \& : $4.2 \times 4.6 \times 3.2 \mathrm{~mm}$. on $R$. ovary, $2 \times 1.5 \times 1.5 \mathrm{~mm}$. near $\mathrm{R}$ kidney. Rabbit $\mathrm{N}$ o : $2.6 \times 1.2 \times 0.9 \mathrm{~mm}$. \& $2.2 \times 1.5 \times 0.9 \mathrm{~mm}$. on $\mathrm{L}$ epididymis, $1.8 \times 1.5 \times 1.2 \mathrm{~mm}$. on $R$ epididymis. Rabbit $\mathrm{O}$ t $: 2.2 \times 2 \times 1.5 \mathrm{~mm}$. $\& 2 \times 2 \times 1 \mathrm{~mm}$. on head of $\mathrm{L}$ epididymis, 2 pieces of $1.3 \times 1.2 \times 1 \mathrm{~mm}$. on $\mathrm{R}$. epididymis. Rabbit $\mathrm{P} \delta: 1 \times 1 \times 0.8$ mm. on front of $\mathrm{L}$ kidney, $3.5 \times 2 \times 3 \mathrm{~mm}$. \& $2.2 \times 1.5 \times 1.5 \mathrm{~mm}$. on L epididymis. Rabbit $\mathrm{Q}$ 우 : 1 piece under $\mathrm{L}$ kidney, $2.2 \times 2 \times 1.5 \mathrm{~mm}$. \& $3.2 \times 2 \times 2 \mathrm{~mm}$. on $\mathrm{L}$ ovary. Rabbit R $\$: 3.5 \times 3 \times 3$ mm., $2 \times 1 \times 1 \mathrm{~mm}$. \& $1 \times 1 \times 1 \mathrm{~mm}$. on L ovary, $2.5 \times 1.5 \times 1 \mathrm{~mm} ., 2.5 \times 2.5 \times 2 \mathrm{~mm}$. \& $1.5 \times 1 \times 1$ mm. on $\mathrm{R}$ ovary. Rabbit $\mathrm{S}$ : $3.5 \times 3 \times 2.5 \mathrm{~mm} ., 2 \times 1.5 \times 1.5 \mathrm{~mm}$. \& $1.5 \times 1 \times 1 \mathrm{~mm}$. on L epididymis, 1 poppy-seed sized on $R$ epididymis.

The above figure clearly shows a remarkable difference in the fatigability of the sciatic-gastrocnemic-preparation in situ between the normal rabbits and the long surviving, doubly decapsulated ones. An individual, Rabbit M, of the latter group makes a single exception. This will be explained below.

Three normal animals $(H \delta, J \hat{\delta}, K \hat{o})$ were capable of responding agamst thythmical electric stimuli applied to the peripheral end of sciatic nerve with contractions of the gastrocnemic muscle for a long duration of time such as 16-49 hours. Contrary to the normal controls, the decapsulated ones gave no more response when stimuli were given interruptedly with regular intervals for 2-8 hours. The differences are decided.

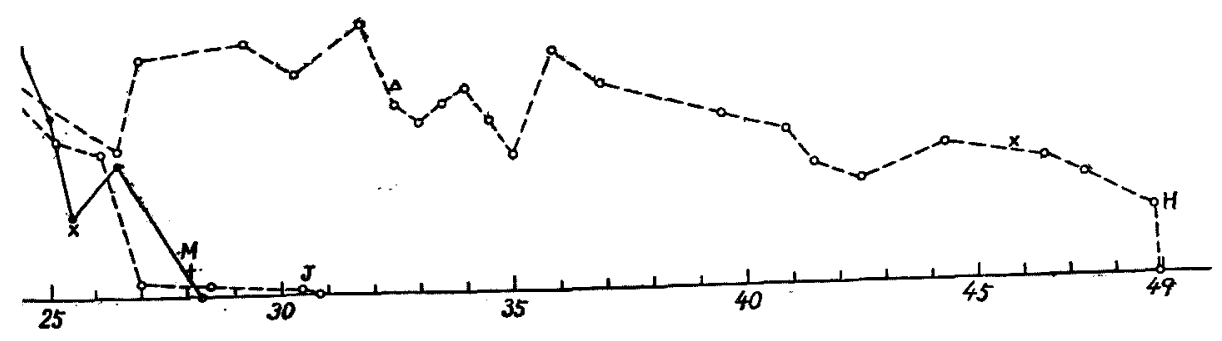


Only Rabbit $M$, doubly decapsulated, behaved just as the normal, 28 hours being noted as needed for the total exhaustion of the nerve-muscle preparation in situ. This was found having possessed a large accessory cortical tissue, such as $4.2 \times 4.6 \times 3.2 \mathrm{~mm}$. on the right ovary, besides an accessory of usual size. This rather abnormally large accessory mass might be taken as responsible for an abnormally large resistance of the nerve-muscle preparation in situ of this rabbit (Rabbit $M$ ).

It may be noted in passing that the contraction height was in some decapsulated animals $(R, S, Q, P \& M)$ was incomparatively great at the start of stimulating in comparision with the normal controls:

On the whole, it may be justifiable to assume that the nerve-muscle preparation of rabbits in situ shows a larger fatigability on the rhythmic stimuli if the suprarenal glands are removed beforehand. The animals were surviving very well the decapsulation.

This conclusion coincides well with the results of the previous experiments above quoted, those of Ferreira and Fontes, ${ }^{6}$ ) who carried out the experiments on the rabbits surviving over two months being excepted.

When they previously conducted the stimulations a few days after double decapsulation, a much quicker fatigability was noted in rabbits; those results check well with what was also based on acute experiments.

The present data, which were performed when the decapsulated rabbits were well, indefinitely surviving, don't' harmonize with the similar kinds of experiment of Ferreira and Fontes, and rather with the data from acute experiments.

The view of Shoji, ${ }^{1)}$ that work capacity of the rabbit is not influenced. by depriving it of the suprarenals, their medulla or the splanchnic nerves, is based on the experiments in which voluntary movements were tested, and the time when fatigue sets in all the kinds of animals was about sixty minutes on an average for each group of animals. The experiments of such a kind are related to the fatigability of the central nervous system, and further the time when the fatigue sets in is undoubtedly shorter than the present experiments; there are therefore no disharmony between the outcome of Shoji's experiment and that in the present investigation.

\section{SUMMARY.}

Of the rapidity with which fatigue develops by ergographic test of the sciatic-gastrocnemic preparation in situ, test was made on the normal rabbits and those which were indefinitely (1-3 months), well surviving after double 
suprarenalectomy. No anesthesia was used, and the body temperature was protected from lowering.

In the so-operated rabbits a complete fatigue sets in much quicker than the normal controls. The velocity with which it develops is about one eighth of that of their controls.

\section{REFERENCES.}

1) Shozi, Tohoku J. of Exp. Med., 1936, 29, 271.

2) Gans and Miley, Am. J. of Physiol., 1927, 82, 1 (Rats, amytal, sciatic stimulation, 1060 minutes in the rats, 1-11 days previously decapsulated, against $8-20 \mathrm{hrs}$. in normal rats.)

3) Kühl, Pfüger Arch., 1927, 215, 277 (Guinea-pigs, gastrocnemius, electrically. 1-2 min. in those, just before decapsulated, $>7-9$ hurs. in the normals.).

4) Ferreira and Fontes, C. r. Soc. Biol., 1928, 98, 987 (Rabbits, chloral hydrate, flexors, faradic stimulation, 5-6 days after double suprarenalectomy, done by the abdominal way. Fatigue quickly developed.).

5) Idd., Arch. Port. d. Sc. Biol., 1929, 2, 236 (The same; this time chloral ether was used.).

6) Idd., C. r. Soc. Biol., 1931, 107, 1167 (The same kind of animal, and the same procedures as before, the rabbits survived over 2 months by improving the method of decapsulation, i.e. by grafting, followed by subsequent removal. The experiment was done under ether. No difference in the fatigability between the normal and the decapsulated.).

7) In gle, Proc. of Soc. for Exp. Biol. \& Med., 1933 (-34), 31, 163 (Rats, sodium luminal, gastrocnemius, faradic stimulation, suprarenal removal by lumbar route, completely fatigued in 15 hrs. (4-33 hrs.), died 2 hrs. later against $>120 \mathrm{hrs.} \mathrm{and} \mathrm{non-lethal.).}$

8) Hales, Haslerud and Ingle, Am. J. of Physiol., 1935, 112, 65 (Detailed report of (7), doubly suprarenalectomized rats worked only for 6-32 hrs., while the control animals worked over 120 hrs. Unilaterally suprarenalectomized rats behaved in quite the same measure as the normal controls.).

9) Ingle, Hales and Haslerud, Am. J. of Physiol., 1935, 113, 200 (Similar experiments as 7-8; the results rê totally suprarenalectomized rats quite the same as before; a close relationship between the amount of suprarenal tissue in partially decapsulated rats and the work performance.).

10) Ingle, Hales and Haslerud, Am. J. of Physiol., 1936, 114, 653. (Similar experiments, autogenous transplants on the surface of ov́ary, no material difference rê work performance between the transplant animals and the normal controls.).

11) Ingle, Am. J. of Physiol., 1936, 116, 622 (Rats, phenobarbital Na, gastrocnemius, $1 \mathrm{hr}$. after double decapsulation. Cortin, subc., increased work capacity, diminished by the ablation.).

12) Ingle, Endocrinology, 1940, 26, 472 (Same experimental conditions, corticosterone acts to increase the work capacity of acutely decapsulated rats.).

13) Ingle, Endocrinology, 1940, 26, 478 (Same experimental conditions as before, work capacity diminishes even if one gland be removed, thus correcting a previous report (8).).

14) Kojima, Tohoku J. of Exp. Med., 1929, 13, 203; O hguri, Ibid., 1931, 17, 390.

15) Sherrington, Mammalian physiology, 1919 Oxford, 110-113.

16) Id., J. of Physiol., 1909, 38, 382. 